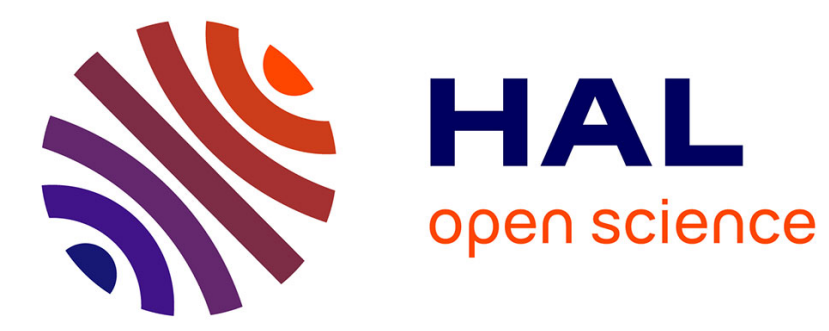

\title{
Spontaneous deformation of hydrogen atom shape in an isotropic environment
}

Roger F. Prat

\section{To cite this version:}

Roger F. Prat. Spontaneous deformation of hydrogen atom shape in an isotropic environment. Journal de Physique, 1988, 49 (4), pp.635-641. 10.1051/jphys:01988004904063500 . jpa-00210737

\section{HAL Id: jpa-00210737 https://hal.science/jpa-00210737}

Submitted on 1 Jan 1988

HAL is a multi-disciplinary open access archive for the deposit and dissemination of scientific research documents, whether they are published or not. The documents may come from teaching and research institutions in France or abroad, or from public or private research centers.
L'archive ouverte pluridisciplinaire HAL, est destinée au dépôt et à la diffusion de documents scientifiques de niveau recherche, publiés ou non, émanant des établissements d'enseignement et de recherche français ou étrangers, des laboratoires publics ou privés. 


\title{
Spontaneous deformation of hydrogen atom shape in an isotropic environment
}

\author{
Roger F. Prat \\ Laboratoire de Photophysique Moléculaire du CNRS (*), Bâtiment 213, Université de Paris-Sud, 91405 Orsay \\ Cedex, France
}

(Reçu le 21 juillet 1987, révisé le 2 novembre 1987, accepté le 9 décembre 1987)

\begin{abstract}
Résumé. - L'énergie potentielle d'un atome d'hydrogène dans un diélectrique est donnée au premier ordre par un terme $V=-\lambda \mathbf{r} .\langle\mathbf{r}\rangle$ fonctionnelle de la densité électronique. Il en résulte une équation de Schrödinger non linéaire contenant le paramètre $\lambda$, de groupe d'invariance $0(3)$. Pourtant, dès que $\lambda>(2 / 9)$ u.a., cette équation a une solution de parité non définie plus stable que l'état $1 s$. La polarisabilité de l'atome dans l'état de parité brisée est nulle en première approximation. Ainsi, il suffit de permettre à l'interaction avec l'environnement de s'adapter à l'état de l'atome pour que la réponse spontanée de ce dernier soit une certaine déformation de sa densité électronique. Ce résultat semble pouvoir être relié à la monovalence de l'espèce «hydrogène atomique ", fait statistique consigné dans la classification périodique.

Abstract. - The potentiel energy of a hydrogen atom in a dielectric medium is given by $V=-\lambda \mathbf{r}$. $\langle\mathbf{r}\rangle$ at first order. This state dependent term yields a parametric non linear Schrödinger equation with spherical symmetry. Yet, a broken parity solution is found to be more stable than the ground $1 s$ state as soon as the parameter $\lambda>(2 / 9)$ a.u. The bifurcated state has zero polarizability in a first approximation. It is shown how the non linear nature of the perturbing potential selects spontaneously a pear-shaped electron density. This result, which is a response of the atom to the interaction with an isotropic environment, is interpreted as depicting the monovalency of atomic hydrogen, a statistical property lying in the periodic classification.
\end{abstract}

A Pierre Claverie, «...toi qui m'as donné du feu quand...»

\section{Introduction.}

In recent years, a growing interest has been given to the molecular structure hypothesis [1] so efficient in explaining the spectra and the symmetry properties of molecules, but strongly conflicting with a quantum mechanical approach avoiding ad hoc hypothesis. No later than last year, Pippard [2] expressed the following view as to the status of theoretical chemistry : «... there is no merit in the claim made occasionnally by physicists, that chemistry is only a branch of physics. Once you realize that no one has yet deduced the structure of the water molecule, $\mathrm{H}_{2} \mathrm{O}$, from first principles, you appreciate how wild that claim is. "Summing up the analysis now prevailing, it is said primarily that orthodox quantum mechanics is not compatible with either the fixednuclei picture or the quasi-localized one, if we require the states arising from them to be eigenstates

(*) Laboratoire associé à l’Université de Paris-Sud. of the total Hamiltonian $H$ for nuclei and electrons in interaction via Coulomb forces; secondly that if states describing the molecular structure as it is are non stationary states of $H$, the problem is [1] : why do they have so long a lifetime ? Finally, ruling out the approximate explanation in terms of heavy and light particles, the observed localization of nuclei could be explained by saying that stationary states of $H$ are so densely close (except may be for some small molecules, e.g. $\mathrm{NH}_{3}$ ) that a natural broadening of levels (due to a coupling with the radiation field [3], medium stabilization [4], ...) is sufficient in order to forbid the observation of the system in an eigenstate of $H$. Molecular spectroscopy would not separate levels within a band width $\Delta E$ wherefrom we are allowed to describe molecules as they are observed in terms of non stationary, but stable states.

Let $H$ be the «molecular» Hamiltonian, for instance the one with fixed nuclei, as it is used in quantum chemistry. The symmetry properties of $H$ versus those possessed by $\mathscr{H}$ stand at the heart of the 
discussion : the invariance group of $H$ is much larger than that of $\mathcal{H}$, so that each irreducible representation (irrep) of the former is generally reducible relative to the latter group. One could say that for almost all molecules, a breaking of symmetry occurs spontaneously in the hamiltonian $H$, giving rise to the molecular Hamiltonian.

1.1 STRUCTURE versus LOCATION. - The underlying localization of nuclei in a molecule naturally induces the deformation of the electron density away from what it would be if the symmetry of $\mathscr{H}$ had been identical with that of $H$ : the electron system acquires a «structure » in a way similar to the one appearing in some nuclei, when they show a rotational spectrum. As for neutral atoms, such a structure does not appear to be stable enough to be observed (if one excepts the excited $\mathrm{He}$ case [5]). If however we test the depth of such a stability, it is found [6] that the lowering from $Z=10$ to $Z=8$ of the nuclear charge in the Hartree-Fock Hamiltonian for a ten electron atomic system produces deformed states in much the same way as in nuclear theory. Note that the parametric potential (in that case the Coulombic attraction) has spherical symmetry for any value of $Z$.

1.2 ATOMIC VAlENCY: A SPECIFIC Response. The entities recombinating in the course of chemical synthesis are most frequently not electrons and bare nuclei, but bound systems, ultimately atoms, «the » particle for chemists. The problem of molecular structure seems to end up at the atomic step, not beyond. That's the reason why we suggest that before a molecular structure appears, there is the birth of an atomic structure in the sense previously discussed. In other words, we guess that there exists particular non stationary states of an atom which, in the infinite set of states a physicist is able to prepare, are those spontaneously set up by the system during its interaction with an environment.

There is a kind of experimental measure for this : the atomic valency. Let us recall what is valency : for chemists, an integer (or several) attached to each atom, and directional properties, specific to each atomic species, both arising from statistics : thousands of molecules obey the periodic classification. When a relatively small variation of the nuclear charge creates a deformed electronic cloud, why wouldn't a kind of interaction with the environment induce a specific deformation of the electron density in an atom, such as at least an homomorphism can be established with the valency property?

1.3 A NON LINEAR SCHRÖDINGER EQUATION FOR VALENCY. - This idea leads quickly to an egg-orhen blindallee: how could such a deformation emerge from the calculations, if not induced by some corresponding anisotropy of the environment (e.g. ligand field theory, or Stark effect, ...) ? Somewhat anticipating, the anisotropy is clearly necessary, because if we think of a spherically symmetric linear operator describing the interaction between the atom and its surroundings, we find stationary states with deformed density (whenever $L \neq 0$ ), but the point is that there is generally no relationship between their directional properties and those afforded by the valency states of the atom (e.g. parity is mostly not conserved in the latter, cf. oxygen, carbon, hydrogen, ...).

If we assume that we take account of the mecium only through some interaction potential acting on the atom, the way-out could be to make it dependent on the state, or better on the electron density of the atom. It leads then to a non linear Schrödinger equation

$$
i \partial_{t} \psi=\left[H_{0}+\lambda f\left(|\psi|^{2}\right)\right] \psi
$$

where $H_{0}$ represents the Hamiltonian of the isolated atomic system, and $\lambda$ is a parameter modulating the strength of the interaction according to medium specificity. The concentration of the atomic species is supposed to be such that one can still speak of the state of an atom in such conditions.

As a consequence of its non linear nature, the equation (1) is likely to provide us, for critical values $\lambda_{c}$ of the $\lambda$ parameter, with the phenomenon of bifurcation. If more specifically we consider the solutions of equation (1)

$$
\psi=\mathrm{e}^{i \omega t} \varphi
$$

corresponding to some real $\omega$, the bifurcation at $\lambda_{\mathrm{c}}$ can happen together with a spontaneous breaking of symmetry $[7,8]$ that is to say that as soon as $\lambda>\lambda_{c}$, the equation (1) may have solutions not belonging to an irrep of the $H_{0}$ 's invariance group, but rather to some irrep of a subgroup of the latter [9]. One should carefully note that the broken symmetry solutions we are interested in are not those with adapted-to- $H_{0}$ symmetry (remind the symmetry breaking in the Hamiltonian, section 1 , for molecules). Furthermore, the subgroup above mentionned is not random among all subgroups : one can foresee a priori the possible subgroup or subgroups. The detailed knowledge of the function $f\left(\left|\psi^{2}\right|\right)$ and of the critical values of $\lambda$ is necessary to decide between these various opportunities [8].

We shall now consider the simplest system : the hydrogen atom in an environment.

\section{The hydrogen atom in an isotropic environment.}

2.1 THE NON LINEAR POTENTIAL FOR HYDROGEN. The model describes the effect of the environment on a collection of hydrogen atoms ; it is reminiscent of the Onsager's one [10]: the medium is a continuum with relative permittivity $\varepsilon$, infinite, homo- 
geneous and isotropic; each hydrogen atom is supposed to be located in a hole, the shapes of which is either spherical or anyway adaptable [11]. Let $\psi$ be the state of some hydrogen atom in situ, a function of time, $\mathbf{r}_{+}$and $\mathbf{r}_{-}$, the position vectors of the proton and of the electron respectively, relative to an origin 0 . We shall assume that with regards to the influence exerted by the atom on the medium, it can be replaced by a mean ideal dipole with a moment :

$$
\begin{aligned}
\boldsymbol{\mu}=\left\langle\psi,\left(\mathbf{r}_{+}-\mathbf{r}_{-}\right) \psi\right\rangle= \\
\quad=\int \psi^{*}\left(\mathbf{r}_{+}, \mathbf{r}_{-}, t\right)\left(\mathbf{r}_{+}-\mathbf{r}_{-}\right) \psi \mathrm{d}_{3} r_{+} \mathrm{d}_{3} r_{-}
\end{aligned}
$$

in atomic units (we shall use atomic units (a.u.) throughout this paper). The reaction field in the cavity due to the dielectric is [18]

$$
\mathbf{E}_{\mathrm{R}}=2 \lambda \boldsymbol{\mu}
$$

where $\lambda$, a positive constant, would be $(\varepsilon-1) /$ $(2 \varepsilon+1) R_{0}^{3}$, would the dipole be a non polarizable one in a spherical hole of radius $R_{0}$. The potential energy of a proton and an electron in this field is $V=-1 / 2 \mathbf{E}_{\mathrm{R}} \cdot\left(\mathbf{r}_{+}-\mathbf{r}_{-}\right)$and we shall limit the expression of the non linear potential to this one, writing

$$
\lambda f\left(\left|\psi^{2}\right|\right)=V=-\lambda \mathbf{r} \cdot\langle\psi, \mathbf{r} \psi\rangle
$$

where $\mathbf{r}=\mathbf{r}_{+}-\mathbf{r}_{-}$. (See discussion below.) Our Schrödinger equation will then look like :

$$
\begin{aligned}
i \partial_{\mathrm{t}} \psi=\left(\frac{p_{+}^{2}}{2 M}+\frac{p_{-}^{2}}{2}-\frac{1}{r}-\lambda \mathbf{r} \cdot\langle\psi\right. & , \mathbf{r} \psi\rangle) \times \\
& \times \psi\left(\mathbf{r}_{+}, \mathbf{r}_{-}, t\right) .
\end{aligned}
$$

As the reaction field is by definition the same wherever is located the cavity in the medium, we are able to separate the center of mass $G$ motion, getting [12], with

$$
\psi\left(\mathbf{r}_{+}, \mathbf{r}_{-}, t\right)=\mathrm{e}^{i \omega t} F(\mathbf{R}) \varphi(\mathbf{r}),
$$

where $(M+1) \mathbf{R}=M \mathbf{r}_{+}+\mathbf{r}_{-}, \omega=E_{\mathrm{c}}+\nu$, the equation for the relative one :

$$
\begin{aligned}
{\left[\left(1 / 2 p^{2} / \mu\right)-\right.} & (1 / r)- \\
& -\lambda \mathbf{r} \cdot\langle\varphi, \mathbf{r} \varphi\rangle] \varphi(\mathbf{r})=\nu \varphi(\mathbf{r}) .
\end{aligned}
$$

We took $\langle F, F\rangle=1$ and wrote $1 / \mu=(1 / M)+1$. Boundary conditions for the solutions $\varphi(\mathbf{r})$ reflect the isotropy of the environment : we can find in the cavity a sphere (center $G$, radius $R_{0}$ ) such that :

$$
\left.\varphi(\mathbf{r})\right|_{r \geqslant R_{0}}=0 \text {. }
$$

The normalization will then be :

$$
\langle\varphi, \varphi\rangle=\int \varphi^{*} \varphi \mathrm{d}_{3} r=1,
$$

where the integration extends to the volume of the sphere $\left(G, R_{0}\right)$.

Before looking at the solutions of equation (2), some comments are necessary.

2.2 Discussion OF THE MODEL. - Although, as far as we know, there has been no similar attempt concerning the valency phenomenon, the functional form we are using for $V$ was proposed earlier by Yomosa [13] who separates solvent from solute variables in a way analogous to the nuclear («slow») and electronic separation in the BornOppenheimer approximation. As formerly mentionned, the choice $V=-\lambda \mathbf{r} \cdot\langle\varphi, \mathbf{r} \varphi\rangle$ is only reminiscent of Onsager's theory because in that view, the potential due to the reaction field would be either $-\lambda r^{2}[14]$, or $-\lambda \mu_{0}^{2}$ [15], respectively energy of two charges in their own reaction field, and of a permanent dipole $\boldsymbol{\mu}_{0}$ in its own $\mathbf{E}_{\mathrm{R}}$. The present formulation needs neither a permanent dipole nor an applied field.

The proposal that we are allowed to condense the effect of the environment on the atom into an interaction term $-1 / 2 \mathbf{r}, \mathbf{E}_{\mathrm{R}}$ would possibly hide the fact that the medium is part of our system. The isotropy properties of the environment induce the spherical symmetry of this interaction potential. This has to be clearly distinguished from the case when the atom is submitted to an electric field $\mathbf{E}$ created by sources not belonging to the system; in which case the interaction term has cylindrical symmetry. The reaction field $\mathbf{E}_{\mathrm{R}}$ does not contain here any dynamical variable of the atom, analogous in this respect to an electric applied field $\mathbf{E}$; however, $\mathbf{E}_{\mathrm{R}}$ is a functional of the atom density so that we have to consider it as a (polar) vector operator for the quantum mechanics of the atom in situ [16].

We can be lead to the functional form $-\lambda \mathbf{r} .\langle\varphi, \mathbf{r} \varphi\rangle$ from another starting point [17], which enlarges considerably its physical content. In this paper, Davies tests the stability of a state under a perturbing potential :

$$
\begin{aligned}
\bar{W}[\psi] & =\sum_{i}\left(\left\langle\psi, \mathbf{r}_{i}^{2} \psi\right\rangle-\left\langle\psi, \mathbf{r}_{i} \psi\right\rangle^{2}\right) \\
& =\sum_{i}\left(\Delta \mathbf{r}_{i}\right)^{2}
\end{aligned}
$$

which describes a fluctuation in the distribution of the position variables of a collection of particles. The first, positive, term will give after variation $\delta \bar{W}$ a linear potential $\sum_{i} r_{i}^{2}$. The second one, on the contrary, will provide us with $-\sum_{i} \mathbf{r}_{i} \cdot\left\langle\psi, \mathbf{r}_{i} \psi\right\rangle$. However, as $\sum_{i}\left\langle\psi, \mathbf{r}_{i} \psi\right\rangle^{2} \neq\left\langle\psi, \sum_{i} \mathbf{r}_{i} \psi\right\rangle^{2}$, the expression obtained does not look like a solvent 
polarization energy [18] as given in Onsager's model. However, if $\psi$ is a Hartree product

$$
\tilde{\psi}=a\left(\mathbf{r}_{1}\right) b\left(\mathbf{r}_{2}\right) \ldots
$$

of orthogonal one particle functions, we have :

$$
\begin{aligned}
\sum_{i}\left(\left\langle\tilde{\psi}, \mathbf{r}_{i}^{2} \tilde{\psi}\right\rangle-\left\langle\tilde{\psi}, \mathbf{r}_{i} \tilde{\psi}\right\rangle^{2}\right) & \\
= & \left\langle\tilde{\psi}, \boldsymbol{\mu}^{2} \tilde{\psi}\right\rangle-\langle\tilde{\psi}, \boldsymbol{\mu} \tilde{\psi}\rangle^{2}
\end{aligned}
$$

where $\boldsymbol{\mu}=\sum_{i} \mathbf{r}_{i}$. The first member is the fluctuation as considered by Davies, the second is - apart from the term $\left\langle\tilde{\psi}, \boldsymbol{\mu}^{2} \tilde{\psi}\right\rangle$ leading to a linear operator the term due to the reaction field.

In the case of atomic hydrogen, there is only one electron the position variable of which is $\mathbf{r}=$ $\mathbf{r}_{+}-\mathbf{r}_{-}$relative to the proton. Then $\bar{W}[\psi]=$ $\left\langle\psi, r^{2} \psi\right\rangle-\langle\psi, \mathbf{r} \psi\rangle^{2}$, where $\psi$ is a Hartree product $F(\mathbf{R}) \varphi(\mathbf{r}) \exp .(i \omega t)$; it is seen that

$$
\bar{W}[\psi]=\bar{W}[\varphi]=\left\langle\varphi, r^{2} \varphi\right\rangle-\langle\varphi, \mathbf{r} \varphi\rangle^{2}=(\Delta \mathbf{r})^{2}
$$

which leads, as explained, to the required $-\lambda \mathbf{r} \cdot\langle\varphi, \mathbf{r} \varphi\rangle$ form.

There is however, in quantum mechanics, a non zero fluctuation of the centre of mass position variable $R$, but, as

$$
\begin{aligned}
\left(\Delta \mathbf{r}_{+}\right)^{2}+\left(\Delta \mathbf{r}_{-}\right)^{2}=2(\Delta \mathbf{R})^{2}+ & (\Delta \mathbf{r})^{2} \times \\
& \times\left(M^{2}+1\right) /(M+1)^{2},
\end{aligned}
$$

it has no consequence on the relative motion.

Lastly, we shall recall [19] that if valency is a response, it is naturally not very sensitive to the precise condition of the environment: cf. the $a$ priori group theoretical knowledge of the symmetry of the bifurcated solutions. In a first step, we limited ourselves to an effective Hamiltonian $\mathrm{H}=\mathrm{H}_{0}+\mathrm{V}$ with just the $V$ necessary to our views : indeed, our $\lambda_{\mathrm{c}}$ values will have to be improved.

\section{Properties of the solutions of equation (2).}

3.1 Any function satisfying.

$$
\left(\frac{p^{2}}{2 \mu}-\frac{1}{r}\right) \varphi_{n l m}=E_{n l}^{0} \varphi_{n l m}(\mathbf{r}),
$$

fulfilling equation (3) and with defined parity is a solution to equation (2), whatever may be the value of $\lambda$ : the reaction field $\mathbf{E}_{\mathrm{R}}$ is then identically zero. These functions $\varphi_{n l m}$ differ from the hydrogenic ones by their radial part $R_{n l}^{\prime}(r)$; they will be called trivial solutions of equation (2).

3.2 SyMmETRY CONSIDERATIONS ( $\lambda$ fixed). - The Hamiltonian in equation (2) is invariant under the group $0(3)$ of rotations and inversions about a point. Let $\varphi$ be a non trivial solution: it has then no defined parity; its symmetry properties are described by the isotropy group $G_{\varphi}$ (a proper or improper subgroup of $0(3)$ leaving $\varphi$ unchanged : $T . \varphi=\varphi, T \in 0(3))$. The orbit of $\varphi$ is the set $\Omega_{\varphi}=\left\{\varphi \varphi^{\prime} \ldots\right\}$ of $\varphi$ and any function $\varphi^{\prime}=T \cdot \varphi$ not strictly equal to $\varphi$ (e.g. $-\varphi$, or $\left.\mathrm{e}^{i \eta} \varphi\right)$. All states in the orbit correspond to the same value of the « eigenvalue» $\nu$ in equation (2). However linear combinations of linearly independent members of the orbit do not correspond to the same $\nu$ value. Therefore, if we look at the shape of the density $|T \cdot \varphi|^{2}$, we find only one shape. That is not customary in linear quantum theory. For instance, consider the $n=2$ excited state of the hydrogen isolated atom; we should be able to draw many isodensity surfaces :

$$
\left|a 2 s+b 2 p_{-1}+c 2 p_{0}+d 2 p_{+1}\right|^{2}=\text { Const. },
$$

given various values to the coefficients $a, b, c, d$. We obtain then many shapes, but essentially four families : the sphere, the "pear ", the «dumb-bell» and the "doughnut" among which only one is not centrosymmetric. The spherical shape would lead to zero valency, the dumb-bell and doughnut ones to divalency, the pear shape to monovalency. When there exists a suitable (isotropic) environment, we shall find that only the pear shape is stable; its isotropy group is $C_{\infty \mathrm{v}}$.

\section{The bifurcation equation.}

Non trivial solutions of equation (2) are not available for arbitrary values of $\lambda$. We shall prove (in a forth coming paper) that the trivial solution $1 s^{\prime}(r)$ will be the unique one as long as the value of $\lambda$ is less than a critical value $\lambda_{\mathrm{c}}$; for media such as $\lambda>\lambda_{\mathrm{c}}$ however, a non trivial solution $\varphi_{1}(\mathbf{r}, \lambda)$ is more stable than $1 s^{\prime}(r)$.

From this it can be inferred [7] that for $0<$ $\lambda-\lambda_{\mathrm{c}}<\eta$ where $\eta$ is a small quantity, the function $\varphi_{1}(\mathbf{r}, \lambda)$ stands close to $1 s^{\prime}(r)$ :

$$
\varphi_{1}(\mathbf{r}, \lambda) \sim 1 s^{\prime}(r)+\left(\lambda-\lambda_{\mathrm{c}}\right) \chi(\mathbf{r}, \lambda)
$$

where $\chi(\mathbf{r}, \lambda)$ has to be different from zero when $\lambda \geqslant \lambda_{\mathrm{c}}$. When the former equation is used in equation (2), it gives $\nu=E_{10}^{0}=E^{0}$ and the linearized (bifurcation) equation, wherein $\chi$ is $\chi\left(\mathbf{r}, \lambda_{\mathrm{c}}\right)$ :

$$
H_{0} \chi-\lambda_{\mathrm{c}} \mathbf{r} 1 s^{\prime} \cdot\left(\left\langle\chi, \mathbf{r} 1 s^{\prime}\right\rangle+\left\langle 1 s^{\prime}, \mathbf{r} \chi\right\rangle\right)=E^{0} \chi .
$$

Somewhat anticipating, we have $\left\langle\chi, 1 s^{\prime}\right\rangle=0$ so that $\varphi_{1}$ is normalized up to second order, and $\chi$ has to be normalizable. The function $\chi$ may be expanded 
on the angular momentum eigenbasis, but only $p$ functions will give a non zero value to the $\lambda_{\mathrm{c}}$-term in equation (5) :

$$
\chi\left(\mathbf{r}, \lambda_{\mathrm{c}}\right)=\sum_{m=-1}^{+1} q_{m}(r, \lambda) Y_{1 m}(\theta, \Phi)
$$

Here $(r, \theta, \Phi)$ refer to a right-handed orthonormal system Gxyz of axes.

4.1 Calculating the Critical value. - Suppose that we know $\chi$; then the real vector

$$
\mathbf{E}(\chi)=\left\langle\chi, \mathbf{r} 1 s^{\prime}\right\rangle+\left\langle 1 s^{\prime}, \mathbf{r} \chi\right\rangle
$$

has a defined direction in $G x y z$, so that we can take that direction for our new $z^{\prime}$ axis. In the system $G x^{\prime} y^{\prime} z^{\prime}$, the $\lambda_{c}$-term in equation (5) would be proportional to $\lambda_{\mathrm{c}} r 1 s^{\prime} \cos . \gamma$. This shows that the required solution may be guessed to be :

$$
\chi\left(\mathbf{r}, \lambda_{\mathrm{c}}\right)=q\left(r, \lambda_{\mathrm{c}}\right) \cos . \gamma
$$

in a suitable reference system $G x^{\prime} y^{\prime} z^{\prime}$. In fact, using the expression equation (6) for $\chi$ in equation (5), we find a system of three coupled equations, which finally amount to a single: equation (7) below is obtained through a change to right-handed $G x^{\prime} y^{\prime} z^{\prime}$ system, which $z^{\prime}$ axis is defined by $\theta^{\prime}=3 \pi / 4, \Phi^{\prime}=1 / 4 \pi$. If $\gamma$ is the angle between $G z^{\prime}$ and the position vector $\mathbf{r}$, we find:

$\cos . \gamma=(1 / 2) \sqrt{2}[\sin . \theta \cos .(\Phi-1 / 4 \pi)-\cos . \theta]$,

so that with

$$
\chi\left(\mathbf{r}, \lambda_{\mathrm{c}}\right)=\sqrt{3 / 2 \pi} q\left(r, \lambda_{\mathrm{c}}\right) \cos . \gamma,
$$

the radial part $q$ appears to be the (unique) convenient solution of the non homogeneous differential equation :

$\hat{A} q-\frac{4 \pi}{3} \lambda_{\mathrm{c}} r 1 s^{\prime}\left(\left\langle 1 s^{\prime}, r q\right\rangle+\left\langle q, r 1 s^{\prime}\right\rangle\right)=E^{0} q$,

where $\hat{A}$ is the radial operator given by the equation :

$$
Y_{1 m} \hat{A} q=H_{0}\left(q Y_{1 m}\right) \text {. }
$$

The scalar products in equation (7) are defined in $L^{2}\left(0, R_{0} ; r^{2} \mathrm{~d} r\right)$ and $1 s^{\prime}$ is the ground state solution of equation (4) normalized according to $4 \pi \int_{0}^{R_{0}}$ $1 s^{\prime 2} r^{2} \mathrm{~d} r=1$. As $q$ in equation (7) is determined only up to a real factor $k\left(\lambda_{\mathrm{c}}\right)$, we define :

$$
\begin{array}{r}
x\left(r, \lambda_{\mathrm{c}}\right)=\left[\frac{4 \pi}{3} \lambda_{\mathrm{c}}\left(\left\langle 1 s^{\prime}, r q\right\rangle+\left\langle q, r 1 s^{\prime}\right\rangle\right)\right]^{-1} \times \\
\times q\left(r, \lambda_{\mathrm{c}}\right) .
\end{array}
$$

Equation (7) is transformed to

$$
\left(\hat{A}-E^{0}\right) x\left(r, \lambda_{\mathrm{c}}\right)=r 1 s^{\prime} .
$$

However, the solution of equation ( $\left.7^{\prime}\right)$ does not depend on the value of $\lambda_{c}$, and is a real function because $E^{0}$ is real; let us call it $x(r)$. Then $q\left(r, \lambda_{\mathrm{c}}\right)$ is in fact the product of a real function of $\lambda_{\mathrm{c}}, k\left(\lambda_{\mathrm{c}}\right)$, by $x(r)$; but $k\left(\lambda_{\mathrm{c}}\right)$ is undetermined and from equation (8), it follows that :

$$
\frac{4 \pi}{3} \lambda_{\mathrm{c}}\left(\left\langle 1 s^{\prime}, r x\right\rangle+\left\langle x, r 1 s^{\prime}\right\rangle\right)=1
$$

from which we find :

$$
\lambda_{\mathrm{c}}=\frac{3}{8 \pi}\left\langle 1 s^{\prime}, r x\right\rangle^{-1} .
$$

To calculate $\lambda_{c}$, we shall assume that the value of $\left\langle 1 s^{\prime}, r x\right\rangle$ is not very different from the value we obtain on using the isolated atom boundary conditions, that is $R_{0}=\infty$. Then $1 s^{\prime} \sim 1 s, E^{0} \sim-1 / 2$ a.u. and $x \sim y(r)$, where $y(r)$ is the solution of the equation :

$$
(\hat{A}+1 / 2) y(r)=\pi^{-1 / 2} r \exp \cdot(-r)
$$

which is $[20]$ :

$$
y(r)=\pi^{-1 / 2}\left(r+1 / 2 r^{2}\right) \exp \cdot(-r) \text { a.u. }
$$

We then find :

$$
\lambda_{\mathrm{c}}=\frac{2}{9} \quad \text { a.u. }
$$

using the formula equation (9).

This result is not surprising. Indeed, we should have been able to write it directly, just after having established that $\mathbf{E}(\chi)$ (therefore $\mathbf{E}_{\mathrm{R}}$ at first order) should have a defined direction: in this case, $\lambda_{\mathrm{c}}$ is formally related to the polarisability $\alpha(=9 / 4$ a.u. $)$ of a hydrogen atom in its ground state by the formula $2 \alpha \lambda_{\mathrm{c}}=1$.

\section{Consequences and conclusion.}

5.1 THE ENERGY NEAR THE BIFURCATION POINT. Equation (2) is derived from a variational principle using the energy functional :

with $\begin{aligned} E[\varphi]=\left(\left\langle\varphi, H_{0} \varphi\right\rangle\right. & \left.-1 / 2 \lambda\langle\varphi, \mathbf{r} \varphi\rangle^{2}\right) \\ \langle\varphi, \varphi\rangle & =1 .\end{aligned}$

Near $\lambda=\lambda_{\mathrm{c}}$, it has the following form (where $\chi$ is $\left.\chi\left(\mathbf{r}, \lambda_{\mathrm{c}}\right)[21]\right)$ :

$$
\begin{aligned}
E[\varphi]= & E^{0}+\left(\lambda-\lambda_{\mathrm{c}}\right)^{2} \times \\
& \times\left[\left\langle\chi,\left(H_{0}-E^{0}\right) \chi\right\rangle-1 / 2 \lambda_{\mathrm{c}} \mathbf{E}^{2}(\chi)\right]+\cdots
\end{aligned}
$$

As $\varphi=1 s^{\prime}$ is a solution to equation (2), there is no 
term of the first degree in $\left(\lambda-\lambda_{c}\right)$. But now, the bracketed quantity in the expression for $E[\varphi]$ may be written

$$
\langle\chi, \chi\rangle\left(W[\chi]-E^{0}\right)
$$

where $W[\chi]$ is that functional from which equation (5) originates when varying $W$. If $\chi$ verifies equation (5) [22], whence $W[\chi]=E^{0}$, so that

$$
E[\varphi]=E^{0}
$$

up to and including second degree terms in $\left(\lambda-\lambda_{c}\right)$. This result may be obtained at once using equations (9), (7') and $\chi(\mathbf{r})=\sqrt{2} x(r) Y_{10}$ in the $G x^{\prime} y^{\prime} z^{\prime}$ system.

5.2 REACTION FIELD AND POLARIZABILITY NEAR $\lambda_{\mathrm{c}}$. - We have found that when $\lambda$ has a value less than $\lambda_{c}=2 / 9$ a.u., the reaction field is a null vector. We do not know what happens when $\lambda \gg \lambda_{\mathrm{c}}$, but a calculation to first order has shown us that

$$
\mathbf{E}_{\mathrm{R}} \sim 2 \lambda_{\mathrm{c}}\left(\lambda-\lambda_{\mathrm{c}}\right) \mathbf{E}(\chi)=\sqrt{6 / \pi}\left(\lambda-\lambda_{\mathrm{c}}\right) \mathbf{e}_{0}^{\prime},
$$

where $\mathbf{e}_{0}^{\prime}$ is the unit vector in the $z^{\prime}$ direction. In other words, everything seems to be as if the hydrogen atom in its cavity were submitted to a uniform electric field (directed along $G z^{\prime}$ ) of value $\sqrt{6 / \pi}\left(\lambda-\lambda_{\mathrm{c}}\right)$. The analogy with a quadratic Stark effect, however, ends up at this point, because the polarizability of our atomic hydrogen in the state $\varphi$ is equal to zero (cf. Eq. (10)).
5.3 THE MEAN INTERACTION POTENTIAL. - With $\langle\varphi, \varphi\rangle=1$ and near $\lambda=\lambda_{\mathrm{c}},\langle V\rangle=-\lambda\langle\varphi, \mathbf{r} \varphi\rangle^{2}$ is given by :

$$
\langle V\rangle=-\frac{3}{2 \pi}\left(\lambda-\lambda_{\mathrm{c}}\right)^{2} / \lambda_{\mathrm{c}}+\cdots
$$

which has a vanishing small value (with $\frac{\lambda-\lambda_{\mathrm{c}}}{\lambda_{\mathrm{c}}}=$ 0.1 , we find $|\langle V\rangle| \sim 0.03 \mathrm{eV})$ : the strength of the interaction is, in our model, linked to the nature of the medium, not to dynamical variables such as pressure, concentration, and so on. If we expressed $\lambda$ as $(\varepsilon-1)(2 \varepsilon+1)^{-1} R_{0}^{-3}$, we should find with $R_{0}=1$ a.u. that the relative permittivity of the dielectric should have to be more than 2.2. As mentionned before, the $\lambda_{c}$ value here calculated through equation (9) is a rough one, as we left aside in the expression for $V$ such terms as $\mathbf{r} \cdot \boldsymbol{\mu} / r^{3}$ in Onsager's model, or as $r^{2}$ in Davies formulation: these will surely alter our $\lambda_{c}$ value.

5.4 CONCLUSION. - The isotropy group of the negative charge density is $C_{\infty \mathrm{v}}\left(z^{\prime}\right)$, which means that the hydrogen atom in its hole digged in an isotropic environment is allowed to deform in one and only one manner, giving rise to a dipolar moment in situ with random orientation (due to an infinite number of points in the orbit $\Omega_{\varphi}$ ). It is that specific response which, in my opinion, accounts for the monovalency of the hydrogen atom.

\section{References}

[1] Wooley, R. G., Isr. J. Chem. 19 (1980) 30. In this issue, see also :

Claverie, P. and Diner, S., p. 54.

[2] Pippard, A. B., Response and stability (Cambridge Univ. Press, Cambridge/London) 1986, p. 208.

[3] Pfeifer, P., Quantum Mechanics in Mathematics, Chemistry and Physics, Eds. K. E. Gustafson and W. P. Reinhart (Plenum, New York) 1981, p. 255.

[4] Bixon, M., Chem. Phys. 70 (1982) 199.

[5] Kelmann, M. E. and Herrick, D. R., J. Phys. B 11 (1978) L 755.

[6] Delgado-Barrio, G. and Prat, R. F., Phys. Rev. $A 12$ (1975) 2288.

[7] SAtTinger, D. H., Topics in Stability and Bifurcation Theory, Springer Math. Lect. Notes 309 (Springer-Verlag, Berlin/New York) 1973.

[8] Michel, L., Rev. Mod. Phys. 52 (1980) 617 and references cited herein.

Constantinescu, D. H., Michel, L. and Radicati, L. A., J. Phys. France 40 (1979) 147.
[9] Cizek, J. and Paldus, J., Phys. Rev. A 3 (1971) 525. Although the authors did not find a bifurcation, they really had one (the symmetry adapted solution should appear for $\lambda>1$ in the Fig. 1 of this paper). Cf. reference [6] above for several observed bifurcations in $\mathrm{O}^{--}$.

[10] Onsager, L., J. Am. Chem. Soc. 58 (1936) 1486.

[11] Rivail, J. L., Terryn, B., Rinaldi, D. and RuizLopeZ, M. F., J. Mol. Struct. 120 (1985) 387.

[12] Bialynicki-Birula, I. and Mycielski, J., Ann. Phys. 100 (1976) 62.

[13] Yomosa, S., J. Phys. Soc. Jpn 44 (1978) 602. I am indebted to $\mathbf{R}$. Constanciel for having called my attention upon this paper.

[14] Hylton, J., Christoffersen, R. E. and Hall, G. G., Chem. Phys. Let. 24 (1974) 501.

[15] Claverie, P. and Jona-Lastnio, G., Phys. Rev. A 33 (1986) 2245.

[16] Merzbacher, E., Quantum Mechanics, 2nd Ed. (John Wiley \& Sons, N.Y./London) 1961, p. 399. 
[17] Davies, E. B., Commun. Math. Phys. 64 (1979) 191.

DAvies, E. B., Irreversible and non-linear dynamics of open systems, in Proc. of NATO ASI, 1979, on Quantum Dynamics of Molecules, Ed. R. G. Wooley (Plenum Press) 1979.

[18] BötTCHER, C. J. F., Theory of Electric Polarization, Vol. 1 (Elsevier Sc. Publ. Co., Amsterdam/London) 1973.

[19] Prat, R. F., Int. J. Quant. Chem. 24 (1983) 697.

[20] Ref. [16], p. 423 and Schulman, J. M., Musher, J. I., J. Chem. Phys. 49 (1968) 4845.
[21] Take $\varphi=\cos \cdot \theta 1 s+\sin \cdot \theta \chi$, calculate $E[\varphi]$ and expand it (using $\lambda=\lambda_{\mathrm{c}}+\lambda-\lambda_{\mathrm{c}}$ and $\theta=\lambda-$ $\left.\lambda_{c}\right)$ up to second order in $\theta$.

[22] Let us denote $u\left(\mathbf{r}, \lambda_{c}\right)$ the first member of equation (5). With $\langle\delta \chi, \chi\rangle=0$ we calculate $\delta W=\langle\chi, \chi\rangle^{-1}[\langle\delta \chi, u\rangle+\langle u, \delta \chi\rangle]$ so that when $W$ is extremal, $u=\alpha \chi$, where $\alpha \in \mathbb{C}$, and $W[\chi]=\operatorname{Re} . \alpha$. However, if $\chi\left(\mathbf{r}, \lambda_{\mathrm{c}}\right) \in \mathbb{R}$ verifies equation (5), then $\alpha=E^{0} \in \mathbb{R}$ and the value of $W$ for that $\chi$ is found to be $E^{0}$. 